University of Montana

ScholarWorks at University of Montana

10-1998

\title{
Asymmetrical Force Production in the Maneuvering Flight of Pigeons
}

Douglas R. Warrick

Kenneth P. Dial

University of Montana - Missoula, kdial@mso.umt.edu

Andrew A. Biewener

Follow this and additional works at: https://scholarworks.umt.edu/biosci_pubs

Part of the Biology Commons

Let us know how access to this document benefits you.

\section{Recommended Citation}

Warrick, Douglas R.; Dial, Kenneth P.; and Biewener, Andrew A., "Asymmetrical Force Production in the Maneuvering Flight of Pigeons" (1998). Biological Sciences Faculty Publications. 216.

https://scholarworks.umt.edu/biosci_pubs/216

This Article is brought to you for free and open access by the Biological Sciences at ScholarWorks at University of Montana. It has been accepted for inclusion in Biological Sciences Faculty Publications by an authorized administrator of ScholarWorks at University of Montana. For more information, please contact

scholarworks@mso.umt.edu. 
The Auk 115(4):916-928, 1998

\title{
ASYMMETRICAL FORCE PRODUCTION IN THE MANEUVERING FLIGHT OF PIGEONS
}

\author{
Douglas R. WARRICK, ${ }^{1,3}$ KenNeTH P. DiAL, ${ }^{1}$ AND ANDREW A. BIEWENER ${ }^{2}$ \\ ${ }^{1}$ Division of Biological Sciences, University of Montana, Missoula, Montana 59812, USA; and \\ ${ }^{2}$ Department of Biology, University of Chicago, Chicago, Illinois 60637, USA
}

\begin{abstract}
Downstroke force produced by Rock Doves (Columba livia) as they negotiated an obstacle course was measured using in vivo recordings of delto-pectoral crest strain. During this slow $\left(<6 \mathrm{~m} \mathrm{~s}^{-1}\right)$, maneuvering flight, pigeons produced a series of four to six successive wingbeats in which the wing on the outside of the turn produced greater peak force than the wing on the inside of the turn, suggesting that the birds maneuvered in a saltatory manner during slow flight. This asymmetrical downstroke force may be used to increase or reestablish bank lost during upstroke, or it may be directed as thrust to compensate for adverse yaw or create excess yaw to alter the bird's direction of flight. Continuous production of asymmetrical downstroke force through a turn differs from the traditional model of maneuvering flight, in which asymmetrical force is used only to initiate a bank, the forces are briefly reversed to arrest the momentum of the roll and then equalized to maintain the established bank, and the redirected lift of the wings then effects a turn. Although this traditional model probably describes most turns initiated during fast and gliding flight in birds, it underestimates the complexity of maneuvering during slow, flapping flight, where sophisticated kinematics and neuromuscular control are needed to change direction effectively. Received 17 July 1997, accepted 4 March 1998.
\end{abstract}

THE GREAT DIVERSITY OF SPECIES within all taxa capable of flight suggests that the behavioral plasticity afforded by flight allows the exploitation of a wide variety of habitats (Norberg 1990). The directness and speed of flight allow great distances to be covered efficiently and quickly such that flying animals can respond to changes in food supply, climatic conditions, or, in more immediate situations, predation pressure. However, speed isn't everything; studies of flight performance (as inferred by external morphology) suggest that the spatial characteristics (e.g. clutter) of a species' habitat exert selective pressure on maneuverability (Norberg 1981, Norberg and Rayner 1987). The inference of maneuvering performance by external flight morphology (e.g. wing loading, aspect ratio) requires a steady-state assumption (i.e. continuous lift production and nonflapping wings). On the contrary, given the enormous variety of bird species that rarely glide, particularly during maneuvering (e.g. small passerines), the vast majority of maneuvering flight must take place during flapping flight. Therefore, our understanding of the relationship between flight morphology and the ecology of the animal must be limited. Further-

\footnotetext{
${ }^{3}$ E-mail: drw@selway.umt.edu
}

more, the proximal physical mechanisms for changing direction in slow, unsteady flight are not understood, which prevents clearly relating this type of maneuvering performance to morphology beyond the requirement of high available mass-specific power.

The mechanisms for changing direction in a steady-state turn are fairly simple: disparate forces produced by the wings cause the bird to roll into a bank (henceforth, an initiating force asymmetry), redirecting lift toward the desired direction of flight. In this type of turn, after a reversal of the initial force asymmetry to halt the rolling momentum of the bird (henceforth, an arresting asymmetry), no further force asymmetry is needed to maintain the bank once it has been established, and the bird turns at a constant rate. The process of changing direction during nonsteady-state (flapping), slow flight is less clear, although presumably it is of critical importance; most birds must be able to maneuver precisely during takeoff and landing. Using two-dimensional light film, Dial and Gatesy (1993) found that pigeons flying at low speed through an obstacle course appear to create a bank using angle-of-attack asymmetries and then fly symmetrically through a turn for several wingbeats. If true, the maneuvering strategy of pigeons in slow flight is the same as 


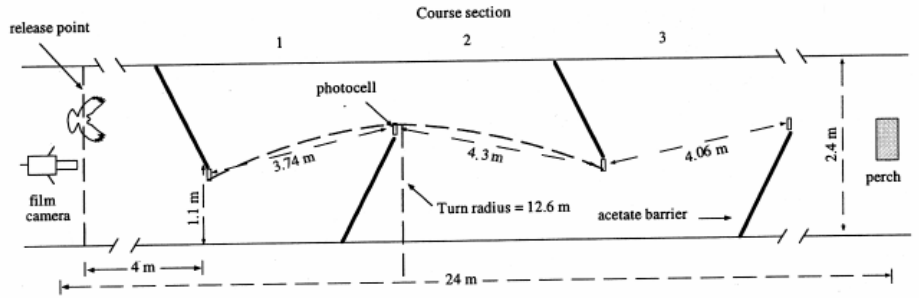

FIG. 1. Schematic diagram of the maneuvering-flight corridor. The turning radius shown is the maximum that would be possible if the pigeons flew "perfectly" (i.e. gliding, constant rate turn, with wings just clearing the acetate barriers). .

that employed in a steady-state turn: they create a bank and allow the redirected lift of downstroke to pull them steadily through the turn. However, kinematic asymmetries may be difficult to discern on two-dimensional film, and small wing asymmetries and changes in yaw and bank may have gone undetected during each wingbeat. Such a slow-flight turn would be produced by a series of small changes in direction (henceforth, a saltatory turn) rather than by establishing a bank and subsequently flying symmetrically through the turn (i.e. a symmetrical turn). The purpose of the present study was to use paired in vivo force recordings of the pectoralis, based on strain recordings of the delto-pectoral crest, to examine the patterns of downstroke force production in pigeons flying through an obstacle course, and thereby determine which turning strategy (symmetrical or saltatory) they employed during slow, maneuvering flight.

\section{MATERIALS AND METHODS}

Bird training, flight corridor, and cinematography.Three Rock Doves (Columba livia; hereafter "pigeons") captured in Missoula, Montana, were trained to fly from the hand through a hallway corridor $(2.5 \times 3 \times 20 \mathrm{~m})$ to a perch. Four clear (to allow

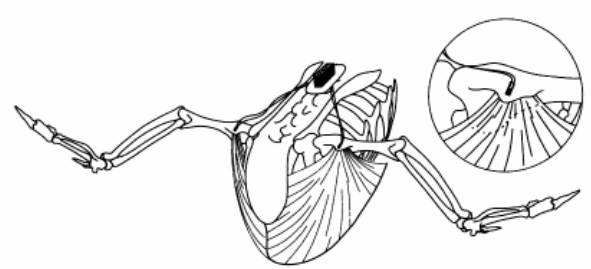

FIG. 2. Attachment site of the strain gauges on the delto-pectoral crests, and back-plug position (inset). the flights to be filmed) acetate barriers ( $1 \mathrm{~m}$ wide $X$ $2.4 \mathrm{~m}$ long, $0.08 \mathrm{~mm}$ thick) were suspended at $4-\mathrm{m}$ intervals within the corridor such that the pigeons needed to make four alternating turns to negotiate the flight course (Fig. 1). The takeoff and landing points were placed far enough from the first and last barriers, respectively, to allow the pigeons to initiate/complete the first and last turns without being influenced by the need to accelerate/decelerate to land. The obstacle course was arranged such that either type of turn (saltatory or symmetrical) was physically possible, with the maximum radius of $12.6 \mathrm{~m}$ for a symmetrical, constant-rate turn around any one of the barriers. Assuming a modest lift coefficient of 1.2 (Norberg 1990), a turn of such radius could be executed by a gliding pigeon in a $45^{\circ}$ bank. During training, the acetate barriers were marked with strips of high-visibility tape to allow the birds to see and learn to avoid the barriers; as the birds became familiar with the course, the tape was gradually removed. The birds were considered ready for experimental trials when they could fly through the entire course without striking the barriers. Four photocells were mounted on the ceiling at the medial edge of each barrier to mark a bird's position as it passed through the assumed middle of each turn. A 16-mm high-speed film camera (RedLake Laboratory Lo-Cam; Kodak 7250 Ektachrome) operating at 150 frames $\mathrm{s}^{-1}$ recorded a posterior view of each trial, $\mathrm{al}-$ lowing two-dimensional kinematic reference and analysis.

Surgical and strain-gauge techniques.-To assess variation in aerodynamic force produced by the wings, we made recordings of the forces experienced by the humerus by attaching a strain gauge to the dorsal surface of the delto-pectoral crest (DPC), which is the prominent anterior tubercle and sole site of pectoralis insertion on the humerus (Fig. 2). Strain recordings from the DPC have provided reliable calibrations of strain-to-force produced by the pectoralis in European Starlings (Sturnus vulgaris), Blackbilled Magpies (Pica pica), and pigeons (Biewener et al. 1992, Dial and Biewener 1993, Dial et al. 1998). For 
purposes here, the force produced by the pectoralis and resultant strain at the DPC can be thought of as the primary antagonist to aerodynamic forces produced by the wing (i.e. a "strut" translating forces from the wing to the body). Thus, we assumed that the pectoralis would reflect the variability in aerodynamic force produced by the wings, regardless of how that variability might have been generated (e.g. changes in area, lift coefficient, or velocity).

Birds were anesthetized ( $25 \mathrm{mg} \mathrm{kg}^{-1}$ ketamine and $2 \mathrm{mg} \mathrm{kg}^{-1}$ xylazine, supplemented as needed) and the feathers removed over both shoulder regions and the middle of the back between the scapulae. On each wing, a 15-mm incision was made in the skin overlying the DPC, which was then exposed by gently parting the fascicles of the deltoid muscle. The dorsal surface of the DPC was prepared by lightly scraping away the periosteum and then swabbing the underlying bone with methyl-ethyl ketone to remove any residual tissue and dry the site. A strain gauge (single-element metal foil type FLE-05-11, Tokyo Sokki Kenkyujo, Ltd.) was then attached to the dorsal surface of the DPC using self-catalyzing cyanoacrylate adhesive, with its principal axis aligned approximately $15^{\circ}$ proximal to perpendicular to the humeral shaft (Dial and Biewener 1993). Strain gauge lead wires (Teflon insulated; Micromeasurements Inc.) ran beneath the deltoid and subcutaneously to a miniature connector plug (12 pole, Microtech FG-6 $[\times 2])$ that was mounted on the back of the bird by suturing the plug's base securely to the intervertebral ligaments using size-O silk thread. The skin was drawn snugly around the protruding connector plug and sutured, and the surrounding skin was then covered with elastic surgical tape.

Strain signals from the DPC were transmitted to bridge amplifiers (Vishay model 2020, Micromeasurements Inc.) via two lightweight $30-\mathrm{m}$ shielded cables. The cables were taped to the bird's back, separated to allow each to pass along either side of the body just anterior to the tail, and then rejoined and draped to the floor. Raw in vivo DPC strains, camerashutter pulse, and photocell signals were then sampled at $5,000 \mathrm{~Hz}$ by a Keithley Instruments A/D converter and stored in a microcomputer. To monitor the condition of the experimental apparatus, live data from each trial were printed on a Gould 2400 chart recorder.

Tensile strains experienced by the DPC during flight through the obstacle course were calibrated to pectoralis force in situ after the flight trials. A heavy nylon thread was tied around the convergent portion of the pectoralis and the pectoral tendon, just below its insertion on the DPC. With the wing held in various degrees of elevation $\left(0^{\circ}, 30^{\circ}, 60^{\circ}\right)$ above horizontal, calibrated forces were applied to the DPC by pulling on the thread with a force transducer in a manner simulating the contraction of the muscle. Calibrations of DPC strain for each position were av- eraged to estimate downstroke force. Recently, a study of three-dimensional wing kinematics showed that pigeons produce force asymmetries during slow, maneuvering flight by creating differential downstroke velocities between the two wings (Warrick and Dial 1998). The initiating asymmetries in downstroke velocity were produced early through mid-downstroke, and the majority $(70 \%)$ of reversing arresting asymmetries were created later in the same downstroke. Because we calibrated the strain gauges in wing-elevation positions of early to mid-downstroke, the asymmetries in peak force were assumed to represent the initiating force asymmetries.

Obstacle course flights, data collection, and analysis.Experimental trials were performed when the birds appeared to be fully recovered from the anesthesia, usually 5 to $10 \mathrm{~h}$ after surgery, and all trials were performed within $24 \mathrm{~h}$. Before we collected data, the birds were allowed several practice flights through the course, beginning with short, straight flights and working up to longer flights and maneuvering around barriers. Once proficiency was attained, each bird flew the course until five complete uncompromised trials (defined as those lacking technical difficulties and/or barrier strikes) were recorded. After the completion of the maneuvering-flight trials, several additional trials were conducted over the same course distance, but with the barriers removed These "straight and level" trials were considered to be measures of typical force asymmetries produced when a bird was not maneuvering in a purposeful manner relative to the obstacle course.

Because of the high sampling rate and the smooth force/strain profile, peak force was considered a reliable representation of downstroke force and was therefore used to describe patterns of force asymmetry (Fig. 3). In all birds, continuous data taken throughout the wingbeat cycle show a disparity in the onset of the increase in the strain/force curves between the right and left sides, beginning in the upstroke portion of the wingbeat cycle. This may have been a result of slight differences in the gauge orientation on the DPC, and/or that the strain gauges were calibrated for strain conditions during the downstroke, rather than the compressive loading characteristic of the upstroke. This asymmetry in onset was consistent within birds; that is, each bird had a side that consistently preceded the other in onset of downstroke strain (suggesting gauge-orientation differences). However, the timing asymmetry is further pronounced in flight modes where upstroke is especially forceful (e.g. takeoff and landing; suggesting gauge compression). Because force production during takeoffs and landings were outside the scope of this study, we excluded them from our analyses of force asymmetry. This timing asymmetry is reduced in all other phases of flight and does not appear to influence the portion of the strain curve (ap- 


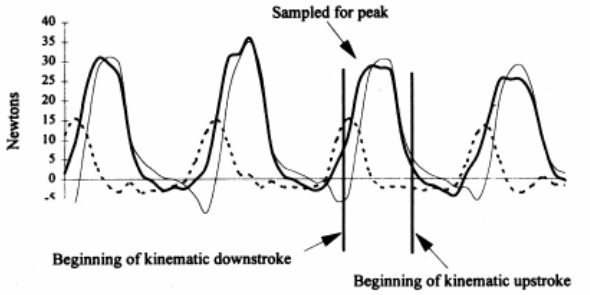

L Left Force $\quad$ Right Force $\quad \cdots \cdots$.... Left - Right Force

FIG. 3. Continuous strain data obtained from Pigeon 1 , collected at $5,000 \mathrm{~Hz}$. Raw strain voltages were calibrated to force in situ. The peak downstroke force (taken from between the vertical lines) was used to represent the force of downstroke and to examine asymmetry patterns. The photocell voltage spike indicates the point at which the bird was passing the medial margin of the barrier.

proximately mid-downstroke) from which peak force was taken.

Kinematic data were collected by projecting ( $\mathrm{L}-\mathrm{W}$ Motion Analyzer Projector model 224-s) the 16-mm film onto a Summagraphics digitizing pad. Using customized digitizing software, bank angle was estimated by the angle between horizontal and a line between the two points where the lateral rectrices insert into the body. Using commercially available software (Dman, Garr Updegraff), peak strain data were extracted for each wingbeat cycle and synchronized to kinematics using the camera-shutter pulse and photocell-trip pulse. Statistical tests were performed using either Microsoft Excel 5.0 or SPSS 6.1.3.

To summarize force-asymmetry patterns, a mean trial for each bird was produced by averaging the bird's force asymmetries over its five maneuveringflight trials. Functional case-equivalent wingbeats were averaged (e.g. the wingbeats in each trial series occurring immediately preceding a barrier, at a barrier, and immediately after a barrier were averaged). In the event that a disparate number of wingbeats occurred in the averaged series, those "extra" wingbeats in series having no functional equivalents were simply included in the summed series as non-averaged cases. Such cases appear as those wingbeats occurring at the midpoint between the barriers.

Expected asymmetries in force production: Two maneuvering flight models.-For a bird maneuvering in accordance with the symmetrical turn model, just prior to the turn an initiating force asymmetry would be produced by the wing on the outside of the turn, creating greater force than the inside wing and causing the bird to roll into a bank (Fig. 4A). An arresting force asymmetry (here, assumed to take place later in the same downstroke) would be needed to halt the

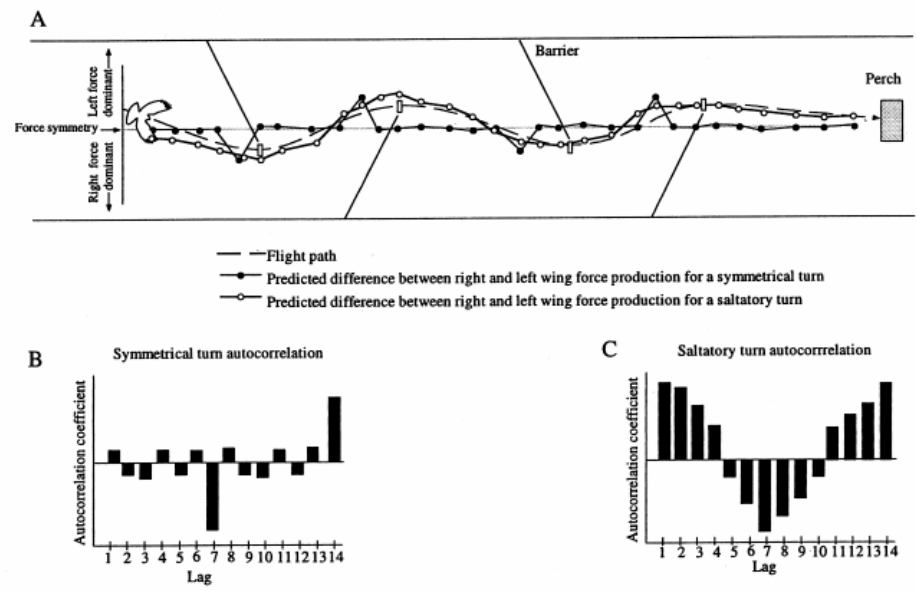

FIG. 4. Predicted pectoralis force asymmetries (A), and asymmetry series autocorrelations for symmetrical (B) and saltatory (C) turns. The autocorrelation coefficient pattern in B was generated by autoregression of a series $n_{i}=0+r_{i}$ with $r_{i}$ equal to a random number selected from a population with an even distribution between 1 and -1 . Every seventh point in the series alternated between $10+r_{i}$ and $-10+r_{i}$, representing the single initiating force asymmetry required for creating banks in either direction (the arresting force asymmetry would generally not be detected by our methodology; see text). The autocorrelation coefficient pattern in (C) was generated by autoregression of a series $n_{i}=\sin \left(n_{g}\right)+r_{i}$, where $n_{g}=n_{g-1}+(360 / 90$; i.e. a "sine wave" force-asymmetry pattern with random variation). 
momentum of the rolling body and stabilize the bird in the desired amount of bank. Once the bank is established, the bird would then need to produce only a series of symmetrical-force wingbeats to effect a turn, thus producing the asymmetry pattern illustrated in Figure 4A. In contrast, a saltatory turn would be created by a series of small force asymmetries in which the wing on the outside of the turn would produce more force than the wing on the inside of the turn (Fig. 4A). Autocorrelation functions of these series of force asymmetries calculated at lags of 1 through 14 are useful in illustrating the differences between these patterns. A highly autocorrelated series of asymmetries would mean that any particular asymmetry in the series would be best predicted by other points in the series. For example, a series with a significant correlation coefficient at a lag of 1 would mean that any point $n$ in the series would be a good predictor of the next point $n+1$ in the series; a significant autocorrelation coefficient at a lag of 2 would mean that a point $n$ in the series would be a good predictor of the point after the next point $n+2$, and so on. The number of lags at which a series is autocorrelated is thus used here as a means of judging the "smoothness" of a series. In a symmetrical turn, the bank-initiating asymmetries would not be highly autocorrelated with the surrounding points in the series (Fig. 4B). In contrast, the saltatory turn model would produce a sinusoidal pattern of force asymmetry, with each force asymmetry in a series being highly autocorrelated with the next (Fig. 4C). This approach was used to test statistically the patterns of asymmetry observed in the force/strain data, and, thereby, determine which of the maneuvering strategies (saltatory or symmetrical) the birds used as they maneuvered through the obstacle course.

\section{RESULTS}

Force production, flight morphology, and general flight characteristics.-The three pigeons used in this study displayed some differences in flight style. Pigeon 1 (wing loading $=59 \mathrm{~N} \mathrm{~m}^{-2}$ ), the least tractable and slowest learner, flew fast $(\bar{x}$ $=5.49 \mathrm{~m} \mathrm{~s}^{-1}$; Fig. 5) and erratically, with occasional high bank angles and frequent speed changes through the course (coefficient of variation $[\mathrm{CV}]$ in speed through the three sections of the course $=0.066$ ). Pigeon 2 (wing loading $\left.=53 \mathrm{~N} \mathrm{~m}^{-2}\right)$ learned quickly and flew fast $(\bar{x}=$ $5.70 \mathrm{~m} \mathrm{~s}^{-1}, \mathrm{CV}=0.043$ ) and dramatically through the course, with some high bank angles. Pigeon 3 (wing loading $=53 \mathrm{~N} \mathrm{~m}^{-2}$ ) also learned quickly, but flew more slowly $(\bar{x}=4.19$ $\mathrm{m} \mathrm{s}^{-1}, \mathrm{CV}=0.053$ ) through the course.

Although not quantified, the birds generally

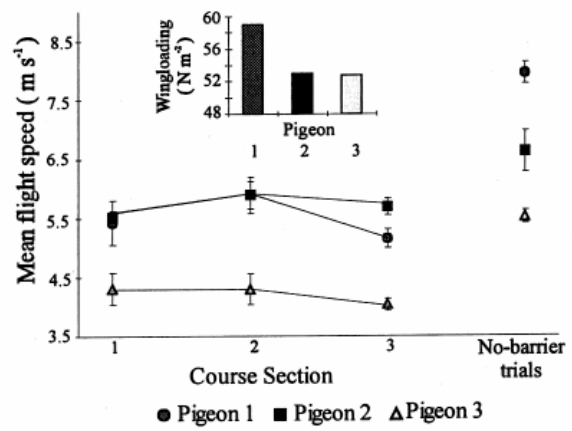

FIG. 5. Wing loadings and flight speeds of the three pigeons through the obstacle course. Flight speeds for the "no-barrier" trials are the average speeds of the pigeons through the portion of the course where the barriers would normally have been situated.

exhibited little variation in altitude as they flew the course, initially climbing to roughly $0.5 \mathrm{~m}$ above the release height of $1 \mathrm{~m}$ and remaining at that height until emerging from the barrier portion of the course. Each bird displayed variability in total peak production of downstroke force (left + right) as they flew the obstacle course, probably reflecting periods of linear and/or angular acceleration and deceleration (Fig. 6). Right and left peak forces were strongly correlated (autoregressive models, $P<$ 0.0001 for all birds in all trials).

Asymmetries in force production: Overall patterns in maneuvering flight.-Consistent with the saltatory model of maneuvering slow flight, a sinusoidal pattern of force asymmetries emerged in many of the obstacle course trials (Figs. 7 and 8). In these trials, the outside wing produced greater force $(\bar{x}=1.58 \mathrm{~N}$, or $5.58 \%$ higher) than the inside wing for an average of 7.15 successive downstrokes. Autocorrelation functions of each trial series of force asymmetries were calculated at lags of 1 through 29 (Fig. 8). Negative coefficients represent the correlation of left dominant force asymmetries (represented as positive values) and right dominant force asymmetries (negative values) used in successive turns in opposite directions. Pigeon 1 flew the most erratically, with only two of its five trials showing significant autocorrelation coefficients at low lags. In other words, any given force asymmetry was poorly predicted by other asymmetries in the series, con- 

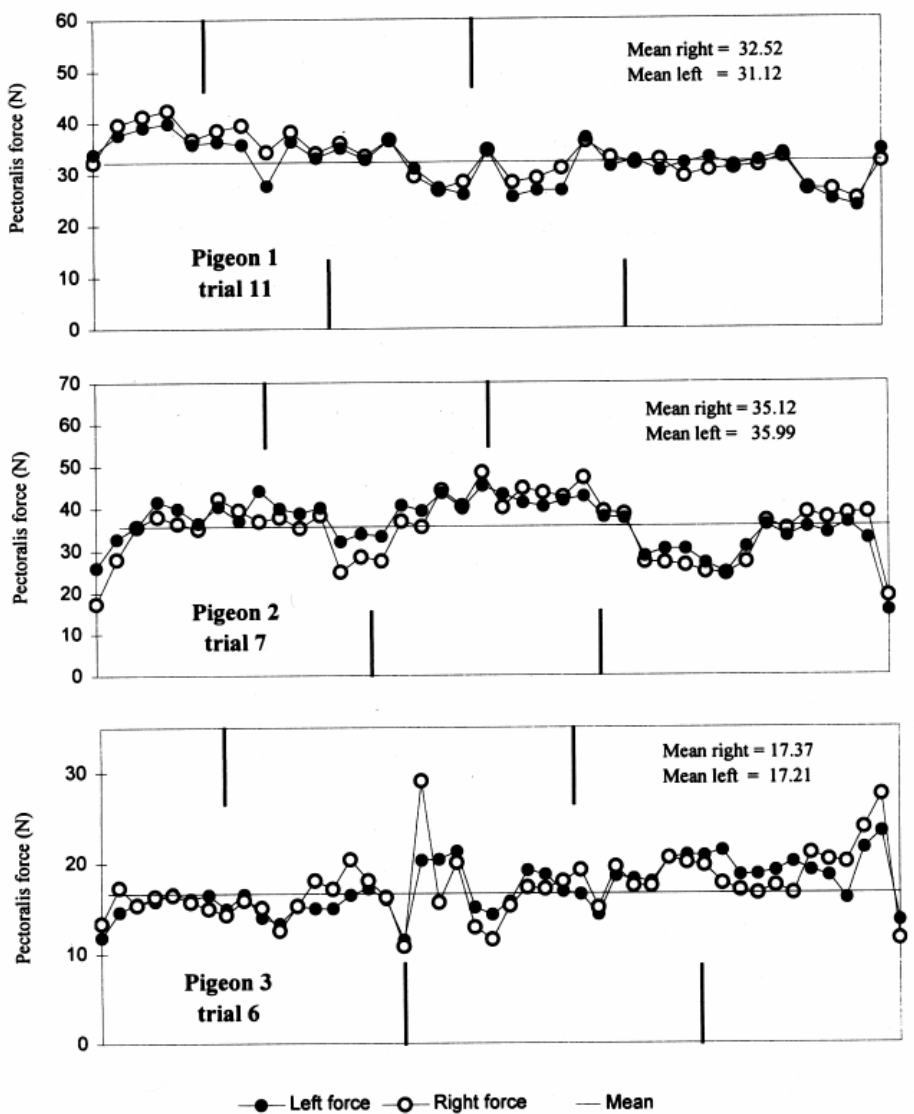

FIG. 6. Peak downstroke forces, right and left wings, of representative trials for each pigeon. The mean force for both wings over the entire run is represented by the horizontal line shown in each panel.

firming a visual impression that there were few strong rhythms in the pattern of force asymmetry (Pigeon 1 in Fig. 8). In contrast, Pigeons 2 and 3 exhibited strong patterns of asymmetry in their bilateral force production. For Pigeon 2, force asymmetries were autocorrelated at low lags in four of five runs, reflecting its studied approach to flying the course (Fig. 8). The patterns of asymmetry produced by Pigeon 3 , though more subtle, were similarly autocorrelated, with three of five trials being significantly autocorrelated at low lags (Fig. 8).

Using autoregressive models, the degree of peak force asymmetry (left minus right) was regressed on the total (left plus right) peak downstroke force to determine: (1) whether changing asymmetries were artifacts of changing total force production (i.e. was greater asymmetry a result of sampling error due to higher total bilateral force production?); and (2) whether the birds were capable of producing subtle force asymmetries during high downstroke force. Peak force asymmetry was not correlated with total peak force production in Pigeon 1 (autoregression coefficient $=0.231$, regression coefficient $=0.030, r^{2}=0.007, P=$ 0.117 ) or Pigeon 2 (autoregression coefficient $=$ 0.170 , regression coefficient $=-0.001, r^{2}=$ $0.0003, P=0.908$ ), and was weakly correlated in Pigeon 3 (autoregression coefficient $=0.200$, regression coefficient $=0.054, r^{2}=0.045, P=$ $0.004)$. 

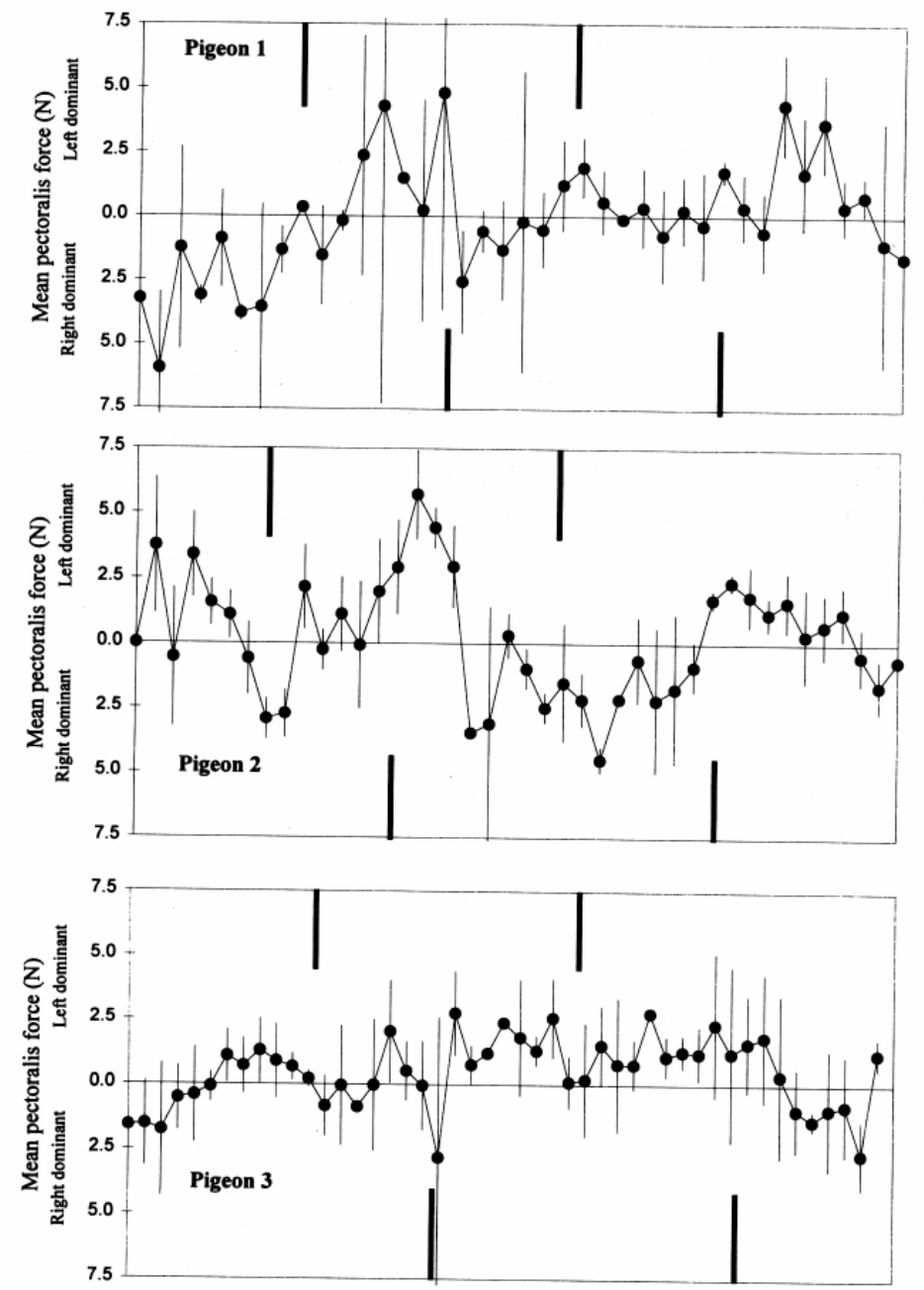

FIG. 7. The "average run" force-asymmetry pattern obtained from each pigeon. Mean force asymmetries were calculated for each case equivalent downstroke asymmetry ( $n=5$ for most; see text). Error bars rep-
resent standard deviations.

Straight and level flight.-Recordings made during straight and level flight illustrate the birds' tendency to produce highly autocorrelated series of slight force asymmetries, but they do not exhibit the sinusoidal asymmetry pattern produced when birds flew the course with barriers (Fig. 9). Force asymmetries during straight and level flight occasionally reflected a "ghost" of the obstacle course, suggesting that birds anticipated maneuvering around barriers that were no longer present. For example, Pigeon 2 produced a large right asymmetry in trial 14 at precisely the point where previously it had needed to maneuver around the first barrier (Fig. 9).

Force asymmetries and body kinematics.-Because the pigeons flew the obstacle course at a body-pitch angle of approximately $30^{\circ}$ to hori- 

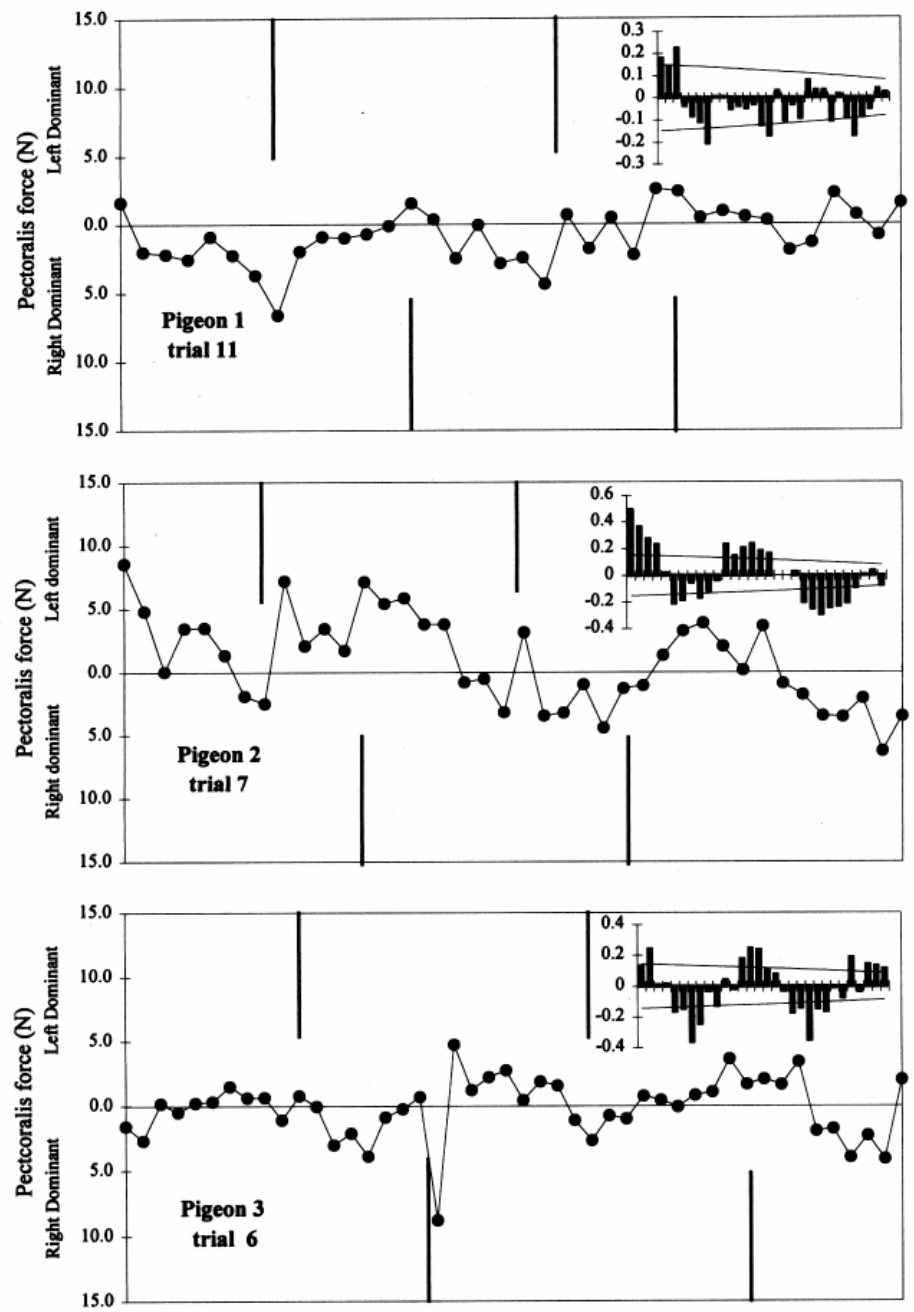

FIG. 8. Three trials best representing each bird's force-asymmetry pattern during maneuvering trials. All birds produced a sinusoidal pattern of force asymmetry in at least some of their trials, although the smoothness of the series was frequently interrupted by a large, dramatic asymmetry (e.g. downstroke 21 in Pigeon 2, trial 7; downstroke 19 in Pigeon 3, trial 6.). Insets show the autocorrelation coefficients at lags of 1 to 30, illustrating the highly autocorrelated nature of the force-asymmetry production. Lines through the autocorrelation coefficients depict $95 \%$ confidence intervals.

zontal, measurements of changes in bank angle during a downstroke are ambiguous; we could not be certain that the observed changes in body orientation were in roll or yaw. Nevertheless, the perceived changes in body angle were taken as coarse measurements of the change in position of the body resulting from the force asymmetry in the downstroke. Occasionally, large changes in body orientation were observed (Fig. 10), illustrating a pigeon's ability to maneuver dramatically when needed, with a change of 60 to $70^{\circ}$ in perceived bank in a single 

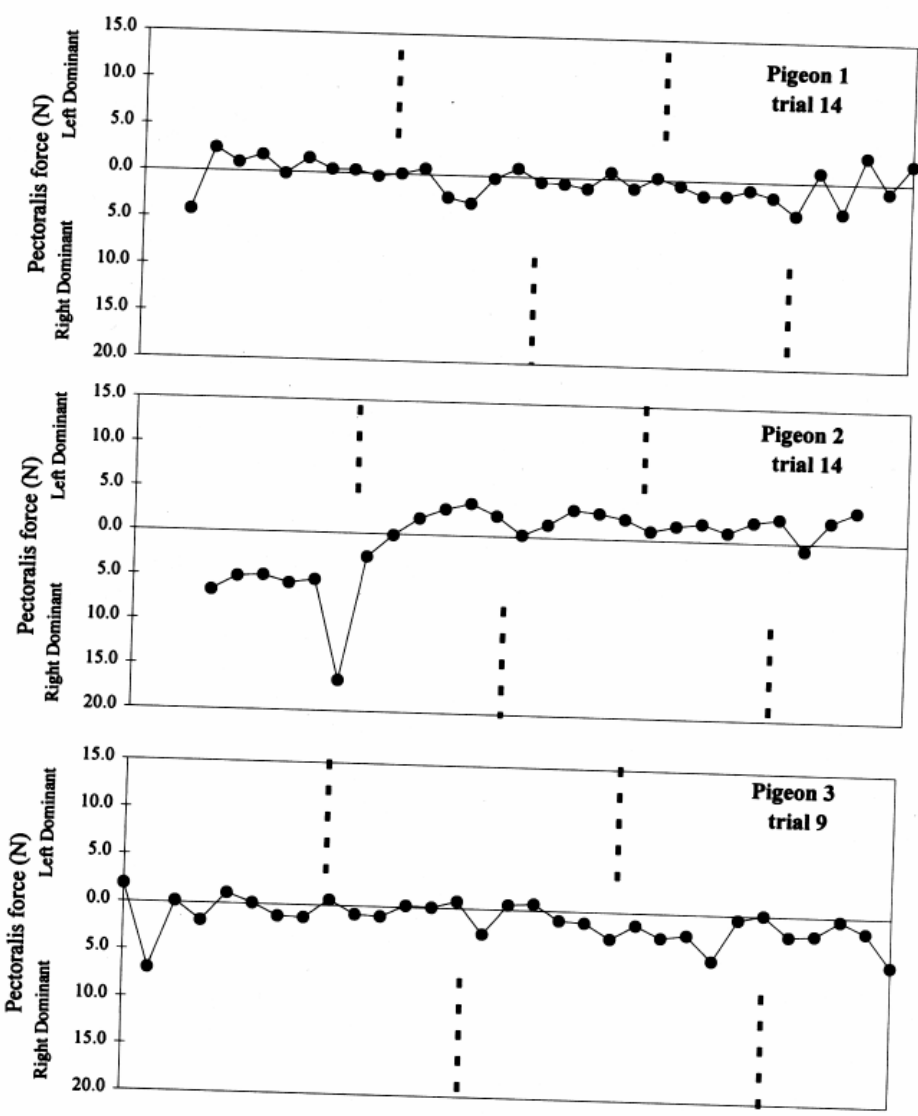

FIG. 9. Force-asymmetry patterns for pigeons during "straight and level" flights. Although highly autocorrelated, these series do not exhibit the sinusoidal pattern of alternating force asymmetry produced dur-
ing the maneuvering trials (see Fig. 8).

downstroke. However, most of the changes in body angle $(79 \%, n=95)$ observed during downstroke were less than $20^{\circ}$ (for all birds; mean right change $=14.6^{\circ}$, mean left change $=$ $16.2^{\circ}$ ).

Aerodynamic force produced by the wings is directed as both lift and thrust, and asymmetry in this latter component would create yawing acceleration. Although we were unable to determine the degree to which the pigeons used lift redirected by bank or yawing mechanisms to effect turns, it is clear that control of yaw can be an important component of maneuvering performance. To use an extreme case as an illustration, Figure 11 depicts Pigeon 1 in an aborted entry into the obstacle course. Rather than continuing down the course, the pigeon executed a climbing turn to the left to reverse its course. The right-dominant asymmetries of the first four wingbeats of the turn (mean right dominance $=12.5 \mathrm{~N}$ ) did not translate into bank; if anything, the bird rolled slightly to the left as the turn progressed. In this instance, the turn during the near-vertical ascent must have been created by a strong yawing movement of the body.

Large force asymmetries also produced high angular accelerations in roll, as illustrated in Figure 10. Such large asymmetries generally occurred only when the birds performed lastinstant, "emergency" maneuvers to avoid the barriers, and usually they resulted in large 


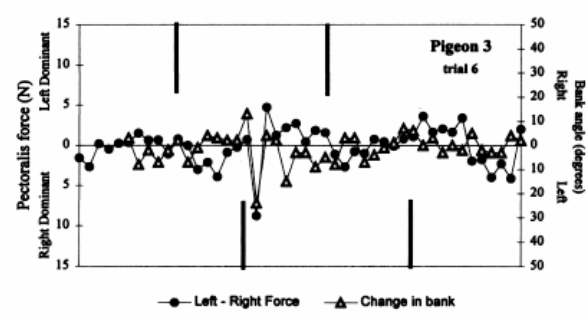

FIG. 10. Pectoralis force asymmetries and the changes in body angle (perceived as "bank"). Body angle was calculated as the two-dimensional angle between a line on the body, drawn from the left side of the insertion of the rectrices to the right side, relative to the horizontal. This angle was recorded at the beginning and end of downstroke. The difference between the angles at the beginning of downstroke and the end represent the change in body angle resulting from that downstroke.

changes in perceived bank angles that persisted for one or two wingbeats (up to $75^{\circ}$; Fig. 10).

\section{DISCUSSION}

Previous electromyographic and kinematic information (Dial and Gatesy 1993) has suggested that pigeons, when flying the same obstacle course that we used, fly symmetrically around the barriers after establishing a bank. Although the force-asymmetry data from our study suggest that pigeons are capable of employing such a strategy, we observed more commonly ( 10 of 15 trials) that the birds produced a series of small force asymmetries to create what we define here as a saltatory turn Changes in body angle resulting from the downstroke corroborate this interpretation of the force asymmetries; that is, few large changes in orientation occurred during the downstroke as the pigeons negotiated a turn The reasons for employing the saltatory strategy are easy to understand: producing small, sequential downstroke asymmetries during each downstroke would be safer than the alternative symmetrical strategy, where the path of the turn would be determined by the execution of one large kinematic event (bank initiation). Conversely, no one kinematic event during a saltatory turn is dramatic, because the bird is making fine positional adjustments ad libitum, compensating for any errors in trajectory that may have resulted from previous downstrokes.
Given a pigeon's ability to produce strong yawing moments (Fig. 11), one might suspect that during saltatory maneuvering the primary axis of rotation would be yaw, which is intrinsically saltatory in nature. Each time the bird produces a change in yaw, its trajectory will change only during the yawing action; as soon as force symmetry is reestablished and the yawing rotation stops, the bird's trajectory will stop changing. Conversely, once a bank is established, the bird's trajectory will continue to change even if it produces symmetrical downstroke forces; to stop changing direction, the bird will need to produce a force asymmetry to level its wings. However, kinematic data indicate that pigeons in slow flight effect a turn primarily by making rapid adjustments in bank angle, and that adverse yaw is created during these changes in bank angle (Warrick and Dial 1998). Adverse yaw initially results from the asymmetry in induced drag as the outside wing increases lift to produce the rolling maneuver, creating yaw in a direction opposite that of the turn. Once the bank is established, some adverse yaw remains, because the wing on the outside of the turn moves through an arc greater than the inside wing and thus has a higher incident airspeed and resultant profile drag.

Because in the present study the two-dimensional perspective results in ambiguity in measuring bank and yaw, we must consider the possibility that the sequential asymmetries we have interpreted as a saltatory turn are not, in fact, force asymmetries developed to compensate for increased drag force on the wing on the outside of the turn. In high-speed flight, adverse yaw can be overcome by twisting the tail (Hoey 1992, Hummel 1992, Thomas 1993, Warrick 1994). In slow flight, however, the tails of the pigeons appeared to be fixed in a flared and bilaterally depressed "flap" configuration, with little or no twisting, presumably augmenting the lift produced by the wings and body (Hummel 1992, Thomas 1993). Presented as such, a tail would not produce any force that could compensate for adverse yaw. Assuming no downstroke-velocity asymmetry and symmetrical wing area (Warrick and Dial 1998), the difference in profile drag between the outside and inside wing would be proportional to the length of the arc each traveled, which, in turn, is proportional to the turn radius for each 


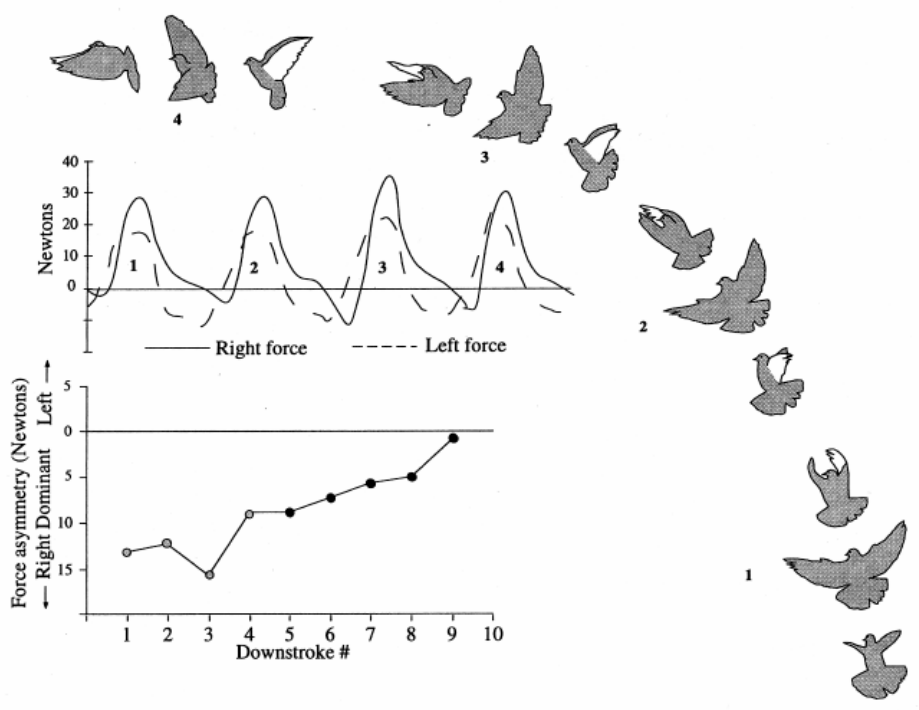

FIG. 11. Serendipitous data collection. In an aborted entry to the obstacle course, Pigeon 1 made an ascending left turn when released. Continuous force data and images (three per downstroke) traced from 150 frame $\mathrm{s}^{-1}$ light film of the first four wingbeats are shown in the upper panel to illustrate how the large forces and force asymmetries produced during downstroke resulted in an ascending, turning flight. The force asymmetries for the first four wingbeats (light markers) and subsequent downstroke force asymmetries as it continued to turn are shown in the lower panel. From the film, appears that most of the angular acceleration the bird produced was around the yaw axis. The radius of this turn would be approximately $1 \mathrm{~m}$.

wing. Assuming a body-path turning radius of $10 \mathrm{~m}$, and given a mean difference in wing-path radius of $0.25 \mathrm{~m}$ (measured as the distance between the wrists at mid-downstroke), the radius of the turn of the outside wing would be $2.5 \%$ larger than that of the inside wing. With a $2.5 \%$ longer arc of travel, the outside wing would thus be traveling $2.5 \%$ faster than the inside at mid-downstroke. Thus, because drag increases as a function of the square of velocity, the outside wing at mid-downstroke would produce $6.25 \%$ more profile drag, assuming equal downstroke velocities. This agrees reasonably well with the $5.58 \%$ mean force asymmetry we observed during turning flight. However, it is unlikely that the strain gauges would detect humeral strains created by increased induced and profile drag force and any increase in pectoralis force that might be used to compensate for these forces (it is unknown if the pectoralis could be used for this task). Data from high-speed $x$-ray film of pigeons flying in a wind tunnel indicate that the DPC is pointed forward, toward the leading edge of the wing's mean chord line-exactly opposite of the direction of the drag force vector (i.e. toward the trailing edge of the mean chord line; K. P. Dial unpubl. data). Thus, the drag-force vector would be perpendicular to the strain gauge's functional orientation, and the gauge would not detect any resulting humeral strains. Furthermore, had the force-asymmetry patterns been a result of drag forces, the "saltatory" pattern of force production would have been seen in every trial (because asymmetrical profile drag would always be created), rather than in only $67 \%$ of the trials.

Clearly, there is more to slow, maneuvering flight than a steady-state model implies. Given the steady-state assumption, maneuverability (defined as turning radius; Norberg and Rayner 1987) has been described purely as a function of wing loading (Pennycuick 1971, Norberg and Rayner 1987), a relationship derived by equating the lift force and the force required to pull an object from a straight path (centripetal force):

$$
1 / 2 \rho C_{L} S v_{w}{ }^{2} \sin \theta=m v_{b}{ }^{2} / r,
$$


where $\theta$ is the bank angle, $\rho$ is the density of air, $C_{L}$ is the dimensionless lift coefficient, $S$ is the area of the wing, $r$ is the radius of the turn, $m$ is the mass of the bird, $v_{w}$ is the velocity of the incident air over the wings, and $v_{b}$ is the velocity of the entire bird. Given the assumption that velocity of the entire bird and the velocity of the wings are equal (i.e. the wings are not flapping), this relationship is generally reduced (e.g. Pennycuick 1971) to the radius of a turn being proportional to wing loading. But birds rarely meet the steady-state assumption of equal velocity of wings and body during maneuvering flight; that is, during most maneuvering, velocity from the centripetal force term $\left(v_{b}\right)$ may be much lower than the velocity of the flapping wings $\left(v_{w}\right)$. Thus, the radius of the turn would be proportional to the ratio of body velocity to wing velocity.

Although steady-state models of maneuvering performance are unable to describe absolute maneuverability in slow flight, they remain a useful measure of what we term intrinsic maneuverability. Birds with lower wing loading are intrinsically more maneuverable, because they are able to perform turns of a smaller radius without flapping than are birds with higher wing loading. Certainly, after including a temporal component to steady-state models, these models should adequately describe the spatial and maneuvering demands on a bird. That is, highly aerial species that spend a lot of time maneuvering during foraging (e.g. swallows) would benefit from the increased efficiency in maneuverability afforded by low wing loading, because they could make small-radius turns without flying slowly and flapping (thus creating a low ratio of body velocity to wing velocity), which would increase the energetic cost of flight (Tucker 1968, 1973; Hails 1979; Goldspink 1981; Hudson and Bernstein 1983; Schmidt-Nielsen 1984). However, when considering the range of spatial habitats that a particular species may use during foraging or nesting, it may be more important to consider its facultative maneuverability, or the absolute limits of a species' ability to produce small-radius turns during flapping flight. This type of maneuvering performance would be a function of the bird's ability to fly slowly (i.e. develop high mass-specific power; Pennycuick 1968, Ellington 1991, Marden 1994) and to change direction during this mode of flight through asymmetrical force production.

The saltatory pattern of force production observed in this study probably represents a locomotor strategy that most birds use during facultative maneuvering, particularly during critical stages of flight such as takeoff and landing. Furthermore, the degree to which the daily routines of most birds depend on creating these controlled locomotor patterns during maneuvering suggests that this ability has been an evolutionary prerequisite ("key innovation;" Liem 1973, Raikow 1986) for the diversification of birds. Although saltatory maneuvering flight performance undoubtedly is a product of a suite of anatomical and physiological features, the production of force asymmetries by the use of asymmetrical downstroke velocities (as in pigeons; Warrick and Dial 1998) suggests that the functional morphology of the primary downstroke muscles (the pectoralis) should be the pretext to any discussion of avian adaptive radiation.

\section{ACKNOWLEDGMENTS}

We thank Randy Trenary, Andi Rodgers, and Will Corning for their help in conducting the experiments. We also thank Abbot Gaunt, Carle Heine, Richard Hutto, Jim Jacobs, and Jerred Seveyka for sparing some thoughts on these matters. Thanks to Bret Tobalske for frequently allowing his morning coffee and evening wine to be spoiled by further discussions. Finally, thanks to Wendy Williams, who walked the first author home. This work was supported by NSF IBN-9211393 and IBN-9507503 to KPD was and performed in accordance with an approved IUCAC protocol (University of Montana's animal assurance \#A3327-01).

\section{LiterATURE Cited}

Biewener, A. A., K. P. Dial, and G. E. Goslow, Jr. 1992. Pectoralis muscle force and power output during flight in the starling. Journal of Experimental Biology 164:1-18.

Dial, K. P., AND A. A. Biewener. 1993. Pectoralis muscle force and power output during different modes of flight in pigeons (Columba livia). Journal of Experimental Biology 176:31-54.

DiAL, K. P., A. A. BIEWENER, B. W. TOBALSKE, AND D. R. WARRICK. 1998. Direct assessment of mechanical power output of a bird in flight. Nature 390: 67-70.

DiAL, K.P., AND S. M. GATESY. 1993. Neuromuscular control and kinematics of the wings and tail 
during maneuvering flight. American Zoologist 33:5.

ELLINGTON, C. P. 1991. Limitations on animal flight performance. Journal of Experimental Biology 160:71-91.

GolDSPINK, G. 1981. The use of muscles during flying, swimming, and running from the point of view of energy saving. Symposium of the Zoological Society of London 48:219-238.

HAILS, C. J. 1979. A comparison of flight energetics in hirundines and other birds. Comparative Biochemistry and Physiology 63A:581-585.

HOEY, R. G. 1992. Research on the stability and control of soaring birds. American Institute of Aeronautics and Astronautics, Inc., Lancaster, California.

HudSON, D. M., AND M. H. BERnSTEIN. 1983. Gas exchange and energy cost of flight in the Whitenecked Raven, Corvus cryptoleucus. Journal of Experimental Biology 13:121-130.

HuMMEL, D. 1992. Aerodynamic investigations on tail effects in birds. Zeitschrift für Flugwissenschaften und Weltraumforschung 16:159-168.

LIEM, K. F. 1973. Evolutionary strategies and morphological innovations: Cichlid pharyngeal jaws. Systematic Zoology 22:425-441.

MARDEN, J. H. 1994. From damselflies to pterosaurs: How burst and sustainable flight performance scale with size. American Journal of Physiology 266:R1077-R1084.

NorberG, U. M. 1981. Flight, morphology and the ecological niche in some birds and bats. Symposium of the Zoological Society of London 48 : 173-197.

NORBERG, U. M. 1990. Vertebrate flight: Mechanics, physiology, morphology, ecology, and evolution. Springer-Verlag, Berlin.

NORBERG, U. M., AND J. M. V. RAYNER. 1987. Ecological morphology and flight in bats (Mammalia; Chiroptera): Wing adaptations, flight performance, foraging strategy and echolocation. Philosophical Transactions of the Royal Society of London Series B 316:335-427.

PENNYCUICK, C. J. 1968. Power requirements for horizontal flight in the pigeon. Journal of Experimental Biology 49:527-555.

PenNYCUICK, C. J. 1971. Gliding flight of the Whitebacked Vulture (Gyps africanus). Journal of Experimental Biology 55:13-38.

RAIKOW, R. J. 1986. Why are there so many kinds of passerine birds? Systematic Zoology 35:255259.

SCHMIDT-NIELSON, K. 1984. Scaling. Why is animal size so important? Cambridge University Press, Cambridge, United Kingdom.

THOMAS, A. L. R. 1993. On the aerodynamics of birds' tails. Philosophical Transactions of the Royal Society of London Series B 340:361-380.

TUCKER, V. A. 1968. Respiratory exchange and evaporative water loss in the flying Budgerigar. Journal of Experimental Biology 48:67-87.

TUCKER, V. A. 1973. Bird metabolism during flight: Evaluation of theory. Journal of Experimental Biology 58:689-709.

WARRICK, D. R. 1994. Flight morphology, performance, and prey capture in a guild of aerial insectivores. American Zoologist 34:5.

WARRICK, D. R., AND K. P. DIAL. 1998. Kinematic, aerodynamic and anatomical mechanisms in the slow, maneuvering flight of pigeons. Journal of Experimental Biology 201:655-672. 